Published in final edited form as:

Proc Int Joint Conf Bioinforma Syst Biol Intell Comput. 2009 January 1; 2009: 185-189. doi:10.1109/

\title{
A Bayesian Approach for Identifying miRNA Targets by Combining Sequence Prediction and Expression Profiling
}

\author{
Hui Liu ${ }^{1}$, Shou-Jiang Gao ${ }^{3,4}$, and Yufei Huang ${ }^{2,4}$ \\ ${ }^{1}$ SIEE, China University of Mining and Technology, Xuzhou, China \\ 2 Department of ECE, University of Texas at San Antonio \\ ${ }^{3}$ Department of Pediatrics, University of Texas Health Science Center at San Antonio \\ ${ }^{4}$ Greehey Children's Cancer Research Institute, University of Texas Health Science Center at \\ San Antonio
}

\begin{abstract}
MicroRNAs (miRNAs) are single-stranded non-coding RNAs shown to plays important regulatory roles in a wide range of biological processes and diseases. The functions and regulatory mechanisms of most of miRNAs are still poorly understood in part because of the difficulty in identifying the miRNA regulatory targets. To this end, computational methods have evolved as important tools for genome-wide target screening. Although considerable work in the past few years has produced many target prediction algorithms, most of them are solely based on sequence, and their accuracy is still poor. In contrast, gene expression profiling from miRNA overexpression experiments can provide additional information about miRNA targets. This paper presents a Bayesian approach to integrate sequence level prediction result with expression profiling to improve the performance of miRNA target identification. The test on proteomic and IP pull-down data demonstrated better performance of the proposed approach.
\end{abstract}

\section{Keywords}

miRNA; Target prediction; Bayesian methods; Gaussian mixtrue model

\section{Introduction}

MicroRNAs (miRNAs) are 19 25 nucleotides non-coding RNAs known to possess important post-transcriptional regulatory functions. It is believed that miRNAs repress translation or cleave mRNA by binding to the complementary sites in the 3'untranslated region (UTR) of the target mRNA, but precise mechanisms underlying their interactions are still not clear. Identifying targets of miRNAs is important for understanding their specific biological functions. Advances have been made in the computational front and past work has produced many target prediction algorithms including TargetScan[1], miRanda[2], PicTar[3] and SVMicrO, and nearly all of them utilize only miRNA-UTR sequence information. All these algorithms still suffer from extremely low prediction precision and sensitivity, and as a result, further improvement is needed.

Since miRNA is known to degrade the target mRNA, genome-wide DNA microarray differential analysis of over-expressing a miRNA can be used to investigate the impact of the miRNA regulation at the mRNA expression level, hence for target prediction. However, the majority of previous research using gene expression profiling for identifying targets have only considered the down-regulated genes as the potential targets. Given that the primary 
function of miRNA is translation inhibition with target mRNA degradation being the secondary mode of regulation, the down-expression of mRNA is clearly neither the sufficient nor the necessary condition for miRNA regulation.

Here, we present a novel algorithm for miRNA target prediction, which combines the sequence binding information with gene expression profiling. In particular, a Bayesian Gaussian mixture model is proposed for modeling the expression profile. To properly model the mixture distribution component of positive targets, the prior distribution constructed based on the existing expression profile of real targets is introduced. Finally, the probability of an mRNA as a target based on the mRNA expression information and the prediction score of its corresponding sequence binding are integrated by a Naïve Bayes model. The algorithm is applied to predict targets for hsa-miR-124, and the prediction performance is evaluated by the IP pull-down and mass spectrometry experiments. Comparison with algorithms based on sequence data alone shows the improved performance of the proposed algorithm.

\section{METHODS}

\section{A. Problem Statement}

For a given mRNA $g$ and a given miRNA $m$, let $t \in\{0,1\}$ denote whether $g$ is a target of $m$. Let $S$ indicate the sequence paring information of $m$ and $g$. Let $e$ represent the differential expression (log fold change) of $g$ due to expressing miRNA $m$. The goal of target prediction is to select most possible value of $t$ base on the expression $e$ and sequence paring $S$. Based on a Naïve Bayes formulation, the desired a posteriori probability (APP) can be calculated as shown in formula (1):

$$
p(t=1 \mid S, e)=\frac{p(e \mid S, t=1) p(t=1 \mid S)}{p(e \mid S)}=\frac{p(e \mid t=1) p(t=1 \mid S)}{p(e)} \propto p(t=1 \mid e) \cdot p(t=1 \mid S)=\alpha(e) \cdot \beta(S)
$$

where the second equality is arrived based on the assumption that $e$ and $S$ are independent, and $\alpha(e)$ and $\beta(S)$ are the APPs of $t$ given $e$ and $S$, respectively, due to the independence between $e$ and $S$. Although $e$ and $S$ are not independent in reality, this assumption reduces the complexity of modeling and the subsequent computation. It has been shown that the Naïve Bayes formulation can achieved satisfactory performance even when the data are correlated. We discuss next the models and approaches for calculating $\alpha(e)$ and $\beta(S)$.

\section{B. Mapping of Sequence Level Prediction Scores to $\beta(S)$}

It is often desirable to take advantage of the existing prediction algorithms including TargetScan and SVMicrO for prediction based on sequence information. These algorithms normally produce a score $s$ for each miRNA-mRNA sequence pairing to indicate the confidence of the mRNA to be a target. To use the prediction of an existing algorithm for our purpose, the score $s$ is assumed to contain all the information of $S$ and $\beta(S)$ can be therefore calculated as $p(t=1 \mid s)$ instead of $p(t=1 \mid S)$. The goal is then to map the score into the $\operatorname{APP} \beta(S)$. To this end, a logistic regression model is used as:

$$
p(t=1 \mid s)=\frac{1}{1+e^{\alpha_{0}+\alpha_{1} s}}
$$

where $\alpha_{0}$ and $\alpha_{1}$ are the parameters to be trained. Particularly, we use the training data constructed for SVMicrO to training the parameters. 


\section{Gaussian Mixture Models of Expression Profile Data}

The gene expression profile of miRNA over-expression experiment contains both the expressions of the positive as well as negative targets. To model the distributions of expression, we first examine the empirical distributions. Figure 1(b) depicts the histogram of the expression levels of 209 experimentally validated targets and that of 11988 measured mRNAs of over-expressing has-miR-124 [4]. Specifically, to obtain the expression of 209 verified targets, we consulted miRecords[5], which collects experimentally verified targets for several species, for human miRNAs and their experimental targets. The expression fold change of each recorded target was retrieved whenever available from the specified miRNA over-expression experiments registered in GEO. Finally, fold change value of 209 verified targets were obtained. Based on these histograms and for computational simplicity, expression data for both positive and negative data are assumed to be Gaussian distributions. Therefore, the genome-wide expression datae is modeled as a mixture Gaussian distribution

$$
\begin{aligned}
p(e \mid \theta) & =p(e \mid t=1) p(t=1)+p(e \mid t=0) p(t=0) \\
& =\mathcal{N}\left(\mu_{+}, \sigma_{+}^{2}\right) \pi_{+}+\mathcal{N}\left(\mu_{-}, \sigma_{-}^{2}\right) \pi_{-} \\
& =\mathscr{M} \mathcal{G}\left(\mu_{+}, \sigma_{+}^{2}, \mu_{-}, \sigma_{-}^{2}, \pi_{+}, \pi_{-}\right)
\end{aligned}
$$

where $\mu, \sigma^{2}$ are the mean and variance of the respective Gaussian mixtures, the subscripts + and - denote the positive $(t=1)$ and negative $(t=0)$ targets, $\pi_{+}+\pi_{-}=1$, and $\theta$ represents the collection of the model parameters. Given model (3), the goal is to uncover mixture components from the expression data, which is equivalent to estimate the parameters from the expression data. Note that since the number of positive targets is only in hundreds, $\pi_{+}$is very small, which means that the component of the positive target is much weaker compared with the negative target and likely to be completely buried in the mixture. This can be illustrated again by Figure 1. Since the positive component is much smaller, the histogram of genome-wide expression for has-miR-124 appears more like a single Gaussian instead of a mixture of two. Unless additional information about the expression of positive data is available, the estimation of the positive component from the mixture is underdetermined and there could be a large number of suboptimal solutions. Fortunately, the expression data of experimentally validated targets are available. These expression levels, although limited in quantity, can be used to aid the estimation of the positive component.

\section{Bayesian Estimation of the Gaussian Mixture}

Under the Bayesian framework, the goal of estimating model parameters $\boldsymbol{\theta}$ is to obtain the posterior distribution

$$
p(\theta \mid e) \propto p(e \mid \theta) p(\theta)
$$

where $p(e \mid \boldsymbol{\theta})$ is the likelihood defined in (3) and $p(\boldsymbol{\theta})$ is the parameter prior distribution. Here, the conjugate priors are adopted and a combination of informative and noninformative priors are defined as

$$
\begin{gathered}
p\left(\mu_{+}, \sigma_{+}^{2} \mid \mathbf{e}_{p}\right)=\mathcal{N} I \mathcal{G}\left(\mu_{N}, \sigma_{+}^{2} / \kappa_{N} ; \alpha_{N}, \beta_{N}\right) \\
p\left(\mu_{-}, \sigma_{-}^{2}\right)=\mathcal{N} \mathcal{I} \mathcal{G}\left(\mu_{0}, \sigma_{-}^{2} / \kappa_{0} ; \alpha_{0}, \beta_{0}\right) \\
p\left(\pi_{+}, \pi_{-}\right)=\operatorname{Dir}\left(\gamma_{+, 0}, \gamma_{-, 0}\right)
\end{gathered}
$$


where $\mathcal{N} \mathcal{I G}$ and Dir are the Normal-Inverse-Gamma and Dirichlet distributions, respectively and $\mathbf{e}_{p}$ denotes the expression profile of the validated targets. It should be clear that an informative prior is applied for the positive component, whereas the noninformative prior is imposed to the negative component. We discuss next the details of these priors. First, the informative $\mathcal{N} \mathcal{I G}$ prior of $p\left(\mu_{+}, \sigma_{+}^{2} \mid e_{p}\right)$ can be obtained from $\mathbf{e}_{p}$ using the standard Bayesian linear Gaussian model by applying a Gaussian likelihood and another noninformative $\mathcal{N} \mathcal{I G}$ prior. Specifically, given the prior of $\mu_{+}$and $\sigma_{+}^{2}$ follows the noninformative $\mathcal{N} \mathcal{I} \mathcal{G}$ distribution

$$
p\left(\mu_{+}, \sigma_{+}^{2}\right)=\mathcal{N} \mathcal{I G}\left(\mu_{0}, \sigma_{+}^{2} / \kappa_{0} ; \alpha_{0}, \beta_{0}\right)
$$

the informative can be shown to be

$$
p\left(\mu_{+}, \sigma_{+}^{2} \mid \mathbf{e}_{\mathbf{p}}\right)=\mathcal{N} I \mathcal{G}\left(\mu_{\mathrm{N}}, \sigma_{+}^{2} / \kappa_{\mathrm{N}} ; \alpha_{\mathrm{N}}, \beta_{\mathrm{N}}\right)
$$

where

$$
\left\{\begin{array}{l}
\mu_{N}=\frac{\kappa_{0}}{\kappa_{0}+N} \mu_{0}+\frac{n}{\kappa_{0}+N} \bar{e} \\
\kappa_{N}=\kappa_{0}+N \\
\alpha_{N}=\alpha_{0}+\frac{1}{2} N \\
\beta_{N}=\beta_{0}+(N-1) s^{2}+\frac{\kappa_{0} N}{\kappa_{0}+N}\left(\bar{e}-\mu_{0}\right)^{2},
\end{array}\right.
$$

$N=209$ in our case, $\bar{e}_{p}$ and $s^{2}$ are the sample mean and variance of $\mathbf{e}_{\mathbf{p}}$, and all other parameters with subscript 0 are the same as those in (5), which define the noninformative prior. Next, for the noninformative priors in (5) and (6), the parameters are chosen as:

$$
\mu_{-}=0, \sigma_{-}=5, \mu_{0}=0, \kappa_{0}=0.2, \alpha_{0}=0.2, \beta_{0}=0.2 \text {. }
$$

Lastly, the parameters of the Dirichlet prior are chosen as $\gamma_{+, 0}=200$ and $\gamma_{-, 0}=20000$, which reflects the common belief that a miRNA regulates about 200 targets[6].

However, since the likelihood assumes the mixture model in (3), the posterior distribution cannot be obtained analytically. A Variational Bayes Expectation Maximization (VBEM) algorithm is applied to estimate the desired distributions.

\section{E. Variational Bayes Expectation Maximization Algorithm}

Since the expression level of each gene is assumed to be i.i.d. and follows the Gaussian mixture (3), the parameters should be estimated from the gene expression profile of all genes $\boldsymbol{e}=\left\{e_{1}, \cdots, e_{G}\right\}$. VBEM algorithm starts by constructing a lower bound on the marginal likelihood function as

$$
\begin{aligned}
& \ln p(e)=\ln \int p(e \mid \pi, \varphi) p(\pi) p(\varphi) d \pi d \varphi \\
& \geq \ln \int q(\pi) \ln \frac{p(e, \pi \mid \varphi)}{q(\pi)}+\ln \frac{p(\varphi)}{q(\varphi)} d \pi d \varphi
\end{aligned}
$$


where as above the inequality is due to the Jensen's inequality, $\boldsymbol{\pi}=\left\{\pi_{+}, \pi_{-}\right\}$,

$\boldsymbol{\varphi}=\left\{\mu_{+}, \mu_{-}, \sigma_{+}^{2}, \sigma_{-}^{2}\right\}$, and $q(\boldsymbol{\pi})$ and $q(\boldsymbol{\varphi})$ are the free distributions introduced to approximate the unknown posterior distributions $p(\boldsymbol{\pi} \mid \boldsymbol{e})$ and $p(\boldsymbol{\varphi} \mid \boldsymbol{e})$ The distributions $q(\cdot)$ (or their parameters) are determined to maximize the lower bound (9). Using the variational derivatives and an iterative coordinate ascent procedure, the optimization can be achieved in an iterative fashion, whose $j+1$ iteration operates as follows:

VBE Step:

$$
q^{j+1}(\pi)=\frac{1}{Z_{\pi}} \exp \left[\int q^{(j)}(\varphi) \ln p(\pi, e \mid \varphi) d \varphi\right]
$$

VBM Step:

$$
q^{j+1}(\varphi)=\frac{1}{Z_{\varphi}} \exp \left[\int q^{(j+1)}(\pi) \ln p(\pi, e \mid \varphi) d \pi\right]
$$

where $Z(\cdot)$ s are the normalizing constants. If $q(\boldsymbol{\pi})$ and $q(\boldsymbol{\varphi})$ are assumed to be the Dirichlet and $\mathcal{N} \mathcal{I G}$ distributions, then (10) and (11) can be obtained analytically. Then, when the algorithm converges, we obtain the approximation to the distributions $p(\pi \mid \boldsymbol{e})$ and $p(\varphi \mid \boldsymbol{e})$ by $q(\boldsymbol{\pi})$ and $q(\boldsymbol{\varphi})$. The MAP or MMSE estimates of $\boldsymbol{\pi}$ and $\boldsymbol{\varphi}$ can be obtained from $q(\boldsymbol{\pi})$ and $q(\boldsymbol{\varphi})$ accordingly.

\section{F. Calculation of $\alpha(e)$}

With the estimated parameters, $\alpha(e)$ can be calculated as

$$
\begin{aligned}
\alpha(e) & =\frac{p(e \mid t=1) p(t=1)}{p(e \mid t=1) p(t=1)+p(e \mid t=0) p(t=0)} \\
& =\frac{\mathcal{N}\left(\hat{\mu}_{+}, \widehat{\sigma}_{+}^{2}\right) \pi_{+}}{\mathscr{M} \mathcal{G}\left(\widehat{\mu}_{+}, \widehat{\sigma}_{+}^{2}, \widehat{\mu}_{-}, \widehat{\sigma}_{-}^{2}, \widehat{\pi}_{+}, \widetilde{\pi}_{-}\right)}
\end{aligned}
$$

where ${ }^{\wedge}$ represents the estimate of the corresponding parameter. An illustration of $\alpha(e)$ is shown in Fig 2. The curve is monotone decreasing in the area of $[-6,0]$, which reflects the existing knowledge that the more significant that an mRNA is down-regulated in the miRNA over-expression experiment, the more likely the mRNA is a real target. However, the curve ramps up for $e>0$, which is because that the negative mixture component has a higher tail than the positive. However, this does agree with the fact that the higher the expression, the unlikely the mRNA is a target. To address this problem, we simply set $\alpha(e)=$ $\alpha(0)$ for $e>0$. This heuristic is simple but works well in practice. Fig. 3 visualizes $\alpha(e)$ with this heuristic.

\section{Result}

\section{A. Sequence Score Retrieval Differential Expression Data}

3'UTR sequences of human were downloaded from UCSC Genome Browser mySQL database. Prediction of genome-wide targets of has-miR-124 using the sequence pairing data were carried out using SVMicrO and the prediction scores were recorded for each mRNA, which were then mapped to the APPs using the function $\beta(S)$. Gene expression profiling of over-expressing has-miR-124 from [4] was then applied and the APPs of each expression 
levels were calculated based on the function $\alpha(e)$ with the heuristic. The integrated score was calculated based on (1) as a product of $\beta(S)$ and $\alpha(e)$.

\section{B. Evaluation using Mass Spectrometry Data}

To evaluate the performance, we first consulted the proteomics data of [7], which measure the protein level of differential expression derived from over-expressing has-miR-124. Since protein inhibition is the primary mode of miRNA silencing, the protein level downexpression should be correlated more directly to the targets than mRNA expression level. As a result, it is of higher confidence to consider the larger down-expressed proteins as real targets. The data consist of the fold change of 1521 proteins. Intuitively, a better prediction algorithm should have higher down-expressed proteins among the top of the prediction ranked by the score. Accordingly, we ranked the prediction algorithms and then examine the cumulative sum of their protein down-regulation in the ranked predictions. Fig. 5 shows the result for the top 50 predictions, which indirectly reflects the prediction precision.

Particularly, the approach "Expression" simply uses mRNA expression as a score and ranks the larger downexpressed gene higher in the list. We note from Fig. 5 that the proposed approach (Combined) achieves the highest amount of protein level down-fold for the top 35 predictions, which indicates higher precision of the proposed approach. The results of different numbers of top predictions for several algorithms are further depicted in Fig 5 . After top 300, the proposed algorithm has the lareget down fold, which also suggests higher sensitive for the proposed algorithm. We conclude based on these results that the proposed algorithm outperforms the sequence-based prediction and prediction based expression data alone.

\section{Precision-Recall (PR) Performance}

Since proteomics data still do not represent the ground true of has-miR-124 targets, further validation is needed. To this end, we adopted the Immunoprecipitation (IP) pull-down according to the scores calculated by each investigated data [4], which measures the potential targets recruited by the ARG-2, an important component of the miRNA effector protein complexes. In this experiment, 388 genes were determined as high confidence targets at a stringent FDR level of 0.01 . We then treated the 388 genes as the true targets of has-miR-124 and investigated the PR performance of different algorithms. The PrecisionRecall curver of proposed algorithm as well as SVMicro and TargetScan were plotted as Figure 6. The result shows a clear enhancement in both precision and recall, when compared to SVMicrO and TargetScan.

\section{Conclusion}

In this paper, we presented a novel algorithm for miRNA target prediction by integrating sequence level prediction result with microarray expression profiling of over-expression miRNA. A Gaussin mixture model was dessigned to model the gene expression profiling and a Bayesian algorithm is deviced to integration the data. The test results on both proteomics and IP pull-down data demonstrated the superior performance of proposed algorithm.

\section{Acknowledgments}

Hui Liu is supported by the project of building high level universities Scholarship Council. Shou-Jiang Gao is supported by NIH grants CA096512 and CA124332. Yufei Huang is supported by an NSF Grant CCF-0546345. 


\section{References}

1. Grimson A, et al. MicroRNA targeting specificity in mammals: determinants beyond seed pairing. Mol Cell 2007;27(1):91-105. S1097-2765(07)00407-8 [pii]. 10.1016/j.molcel.2007.06.017 [PubMed: 17612493]

2. Enright AJ, et al. MicroRNA targets in Drosophila. Genome Biol 2003;5(1):R1. [pii]. 10.1186/ gb-2003-5-1-r1gb-2003-5-1-r1 [PubMed: 14709173]

3. DaW Grun YL, Langenberger D, Gunsalus KC, Rajewsky N. microRNA target predictions across seven Drosophila species and comparison to mammalian targets. PLoS Comput Biol 2005;1:e13. [PubMed: 16103902]

4. Hendrickson DG, et al. Systematic identification of mRNAs recruited to argonaute 2 by specific microRNAs and corresponding changes in transcript abundance. PLoS ONE 2008;3(5):e2126.10.1371/journal.pone.0002126 [PubMed: 18461144]

5. Xiao F, et al. miRecords: an integrated resource for microRNA-target interactions. Nucleic Acids Res. 2008 gkn851 [pii]. 10.1093/nar/gkn851

6. Krek, Azra. Combinatorial microRNA target predictions. Nature Genetics 2005;37:495-500. [PubMed: 15806104]

7. Baek D, et al. The impact of microRNAs on protein output. Nature 2008;455(7209):64-71. nature07242 [pii]. 10.1038/nature07242 [PubMed: 18668037] 


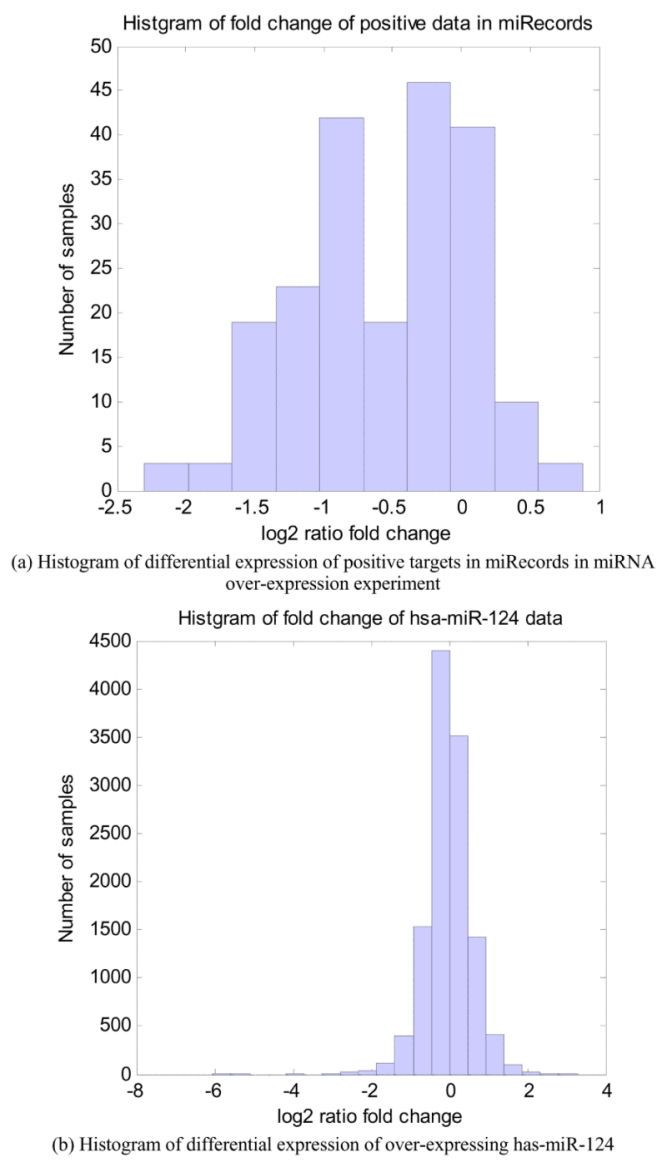

Figure 1.

Histograms of Gene Expression Profiles 


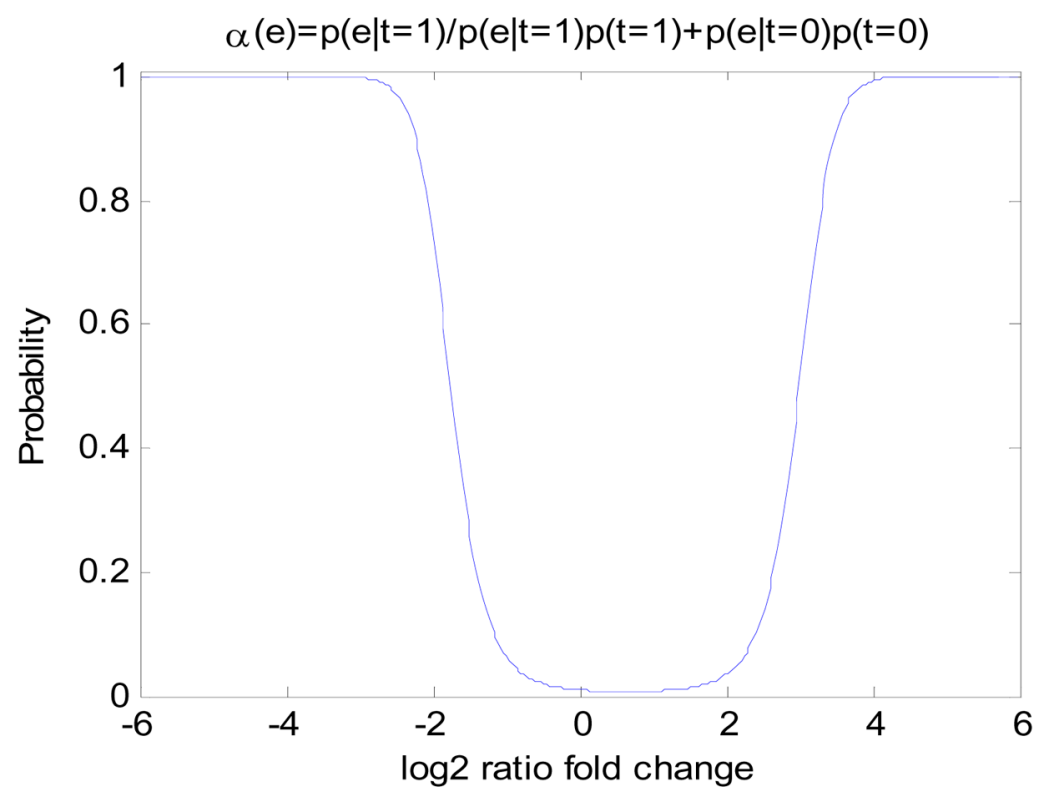

Figure 2.

Plot of $\alpha(e)$ 


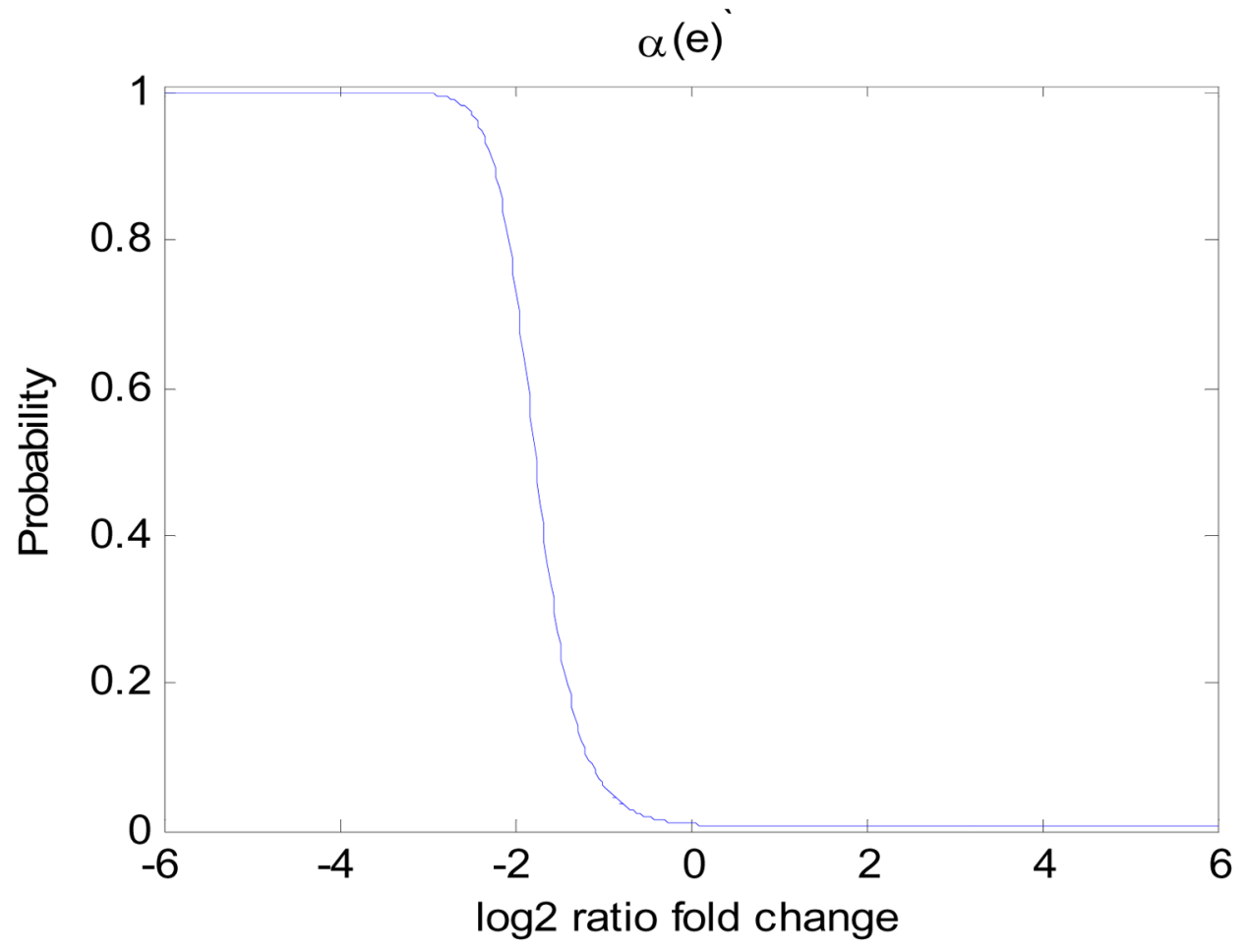

Figure 3.

Plot of $\alpha(e)$ with the heuristic 


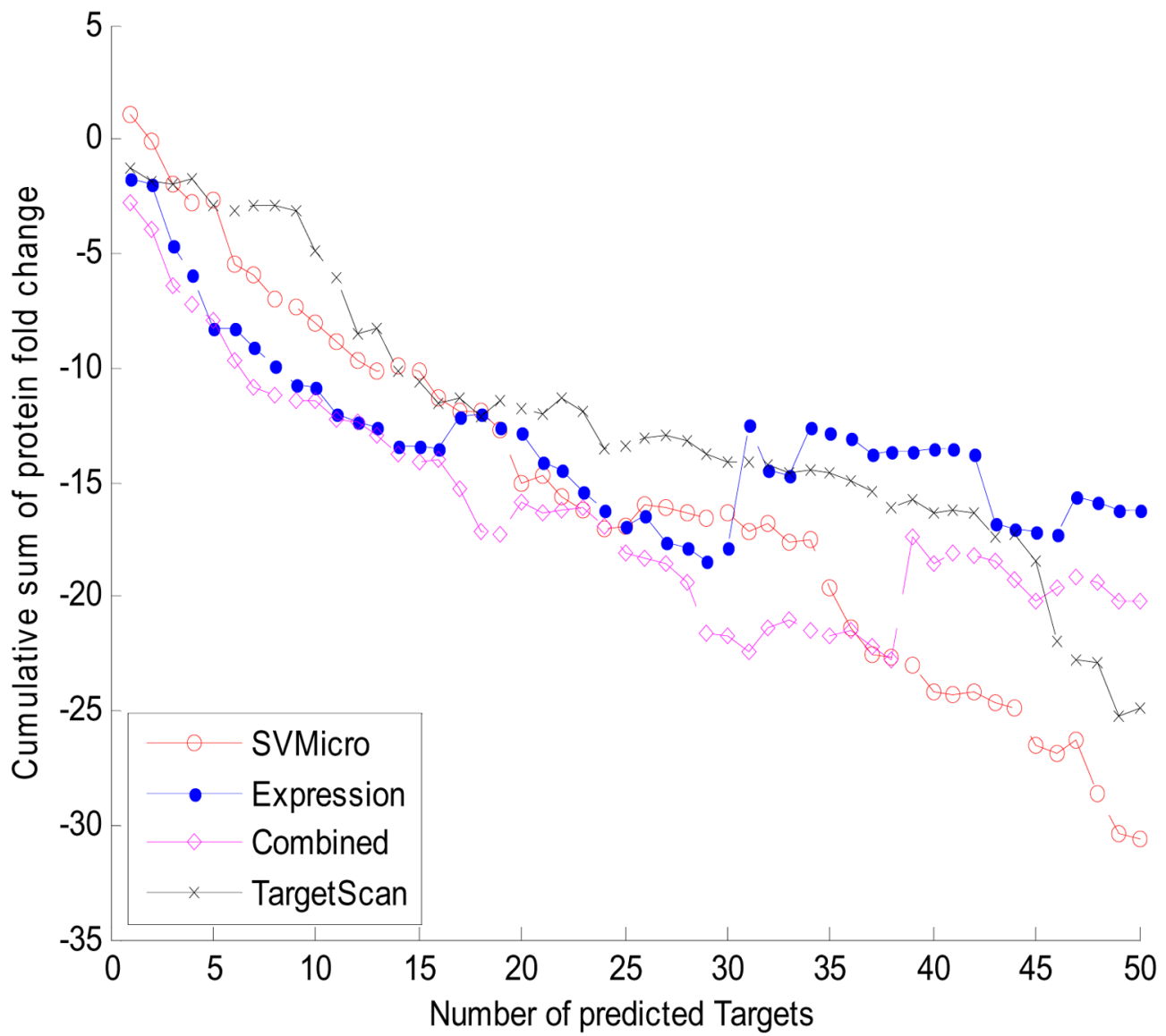

Figure 4.

Cumulative Sum of Protein Fold Change of top 50 predictions. 


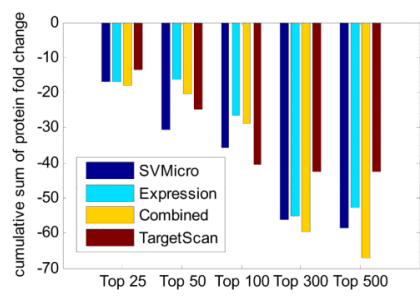

Figure 5.

Cumulative Sum of Protein Fold Change 


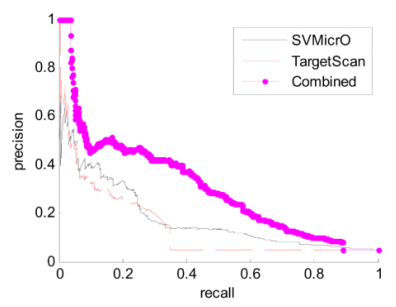

Figure 6.

Precision Recall Curve Based on IP Pull-down Data 